\title{
Intensive Reasoning Training Alters Patterns of Brain Connectivity at Rest
}

\author{
Allyson P. Mackey, ${ }^{1}$ Alison T. Miller Singley, ${ }^{2}$ and Silvia A. Bunge ${ }^{1,2}$ \\ ${ }^{1}$ Helen Wills Neuroscience Institute and ${ }^{2}$ Department of Psychology, University of California at Berkeley, Berkeley, California 94720
}

Patterns of correlated activity among brain regions reflect functionally relevant networks that are widely assumed to be stable over time. We hypothesized that if these correlations reflect the prior history of coactivation of brain regions, then a marked shift in cognition could alter the strength of coupling between these regions. We sought to test whether intensive reasoning training in humans would result in tighter coupling among regions in the lateral frontoparietal network, as measured with resting-state fMRI (rs-fMRI). Rather than designing an artificial training program, we studied individuals who were preparing for a standardized test that places heavy demands on relational reasoning, the Law School Admissions Test (LSAT). LSAT questions require test takers to group or sequence items according to a set of complex rules. We recruited young adults who were enrolled in an LSAT course that offers $70 \mathrm{~h}$ of reasoning instruction $(n=$ $25)$, and age- and IQ-matched controls intending to take the LSAT in the future $(n=24)$. rs-fMRI data were collected for all subjects during two scanning sessions separated by $90 \mathrm{~d}$. An analysis of pairwise correlations between brain regions implicated in reasoning showed that fronto-parietal connections were strengthened, along with parietal-striatal connections. These findings provide strong evidence for neural plasticity at the level of large-scale networks supporting high-level cognition.

\section{Introduction}

Correlations in spontaneous fluctuations of blood oxygenation level-dependent (BOLD) fMRI signal at rest are thought to reflect the prior history of coactivation of brain regions (Dosenbach et al., 2007; Seeley et al., 2007; Fair et al., 2009). These correlations have been interpreted as stable markers of an individual's neural functioning (Fox and Raichle, 2007; Shehzad et al., 2009). However, if correlations do reflect a prior history of coactivation, then they should be influenced by experience. While there is ample evidence of experience-dependent plasticity at the cellular level (Fu and Zuo, 2011), there is little evidence of plasticity in largescale functional networks in humans. The timing of the changeability of rs-fMRI networks is unknown, but it is reasonable to suppose that structural changes are accompanied by, or even preceded by, changes in patterns of functional activity. In other words, examining experience-dependent changes in resting-state connectivity may provide a window into network plasticity in humans that could complement evidence from structural imaging studies.

\footnotetext{
Received Aug. 29, 2012; revised Dec. 9, 2012; accepted Jan. 7, 2013.

Author contributions: A.P.M. and S.A.B. designed research; A.P.M. and A.T.M.S. performed research; A.P.M. analyzed data; A.P.M. and S.A.B. wrote the paper.

A.P.M. was supported by a National Science Foundation Graduate Research Fellowship, and the research was supported by an NINDS Program Project Grant (NS040813, Lead Principal Investigator: M. D'Esposito; Co-investigator: S. A. Bunge). We thank Chloe Green, Talia Seider, Brendan Berry, Joshua Hoerger, and Sarah Inkelis for help with behavioral assessments, and Kirstie Whitaker, Ariel Rokem, Elizabeth Mormino, Carter Wendelken, Matthew Brett, Jean-Baptiste Poline, and Miles Lopes for helpful discussions about data analysis. We also thank Blueprint Test Preparation for their assistance with recruitment and obtaining practice test scores.

The authors declare no competing financial interests.

Correspondence should be addressed to Allyson P. Mackey, Helen Wills Neuroscience Institute, 132 Barker Hall, University of California at Berkeley, Berkeley, CA, 94720.E-mail: a_mackey@berkeley.edu.

DOI:10.1523/JNEUROSCI.4141-12.2013

Copyright $\odot 2013$ the authors $\quad 0270-6474 / 13 / 334796-08 \$ 15.00 / 0$
}

The aim of the present study is to investigate changes in resting-state connectivity associated with training on relational reasoning, or the ability to compare and combine mental representations (Halford and Wilson, 1998; Hummel and Holyoak, 2005). Many brain regions are engaged during reasoning, including frontal and parietal cortices and the striatum (Melrose et al., 2007; Ferrer et al., 2009; Krawczyk, 2010; Prado et al., 2011). Among these regions, we consider that some are integral to relational processing, whereas others support task performance through more general roles in working memory, cognitive control, and/or motor control. Our laboratory has proposed that relations between stimuli are maintained in working memory by lateral parietal cortex, along with domain-specific regions, and then compared or integrated by rostrolateral prefrontal cortex (RLPFC) (Wendelken et al., 2011, 2012). Broadly consistent with this hypothesis, RLPFC and lateral parietal cortex exhibit strong temporal correlations during reasoning (Wendelken and Bunge, 2010; Wendelken et al., 2012) and at rest (Nelson et al., 2010; Mars et al., 2011).

Here, we sought to test the hypothesis that experience with reasoning tasks would lead to tighter coupling between lateral parietal cortex and RLPFC. Additionally, we explored whether reasoning training would alter connectivity among the broader set of brain regions that are commonly engaged during reasoning tasks. Rather than designing a laboratory-based training paradigm, we studied the effects of participating in a 3-month course designed to prepare aspiring lawyers for the Law School Admission Test (LSAT), a standardized test that places strong demands on reasoning (see also Mackey et al., 2012). We selected this course knowing both that high levels of motivation and increased dopamine levels are associated with greater neuroplasticity in adult animals (Bao et al., 2001; Bergan et al., 2005) and that 
Table 1. Participant information

\begin{tabular}{llc}
\hline & Trained & Control \\
& $N=25$ & $N=23$ \\
\hline Age & $22.15(1.88)$ & $21.49(2.03)$ \\
WASI matrix & $29.04(2.49)$ & $29.74(1.74)$ \\
WASI vocabulary & $66.04(5.79)$ & $67(3.71)$ \\
Days between scans & $90.48(16.32)$ & $92.09(23.16)$ \\
Perceived stress & & \\
$\quad$ Time 1 & $21.86(5.04)$ & $20.48(8.45)$ \\
$\quad$ Time 2 & $21.88(6.19)$ & $21.48(8.90)$ \\
Hours of sleep & & \\
$\quad$ Time 1 & $7.52(0.81)$ & $7.61(0.93)$ \\
$\quad$ Time 2 & $7.35(0.99)$ & $7.35(1.16)$ \\
\hline
\end{tabular}

Means and SDs are reported for the variables that the trained group and control group were matched on ( $P$ values $>0.2$ ). Raw scores are reported for the WASI subscales.

students are highly motivated to study for the LSAT because it determines the caliber of law school to which they can gain acceptance. Further, because more than 100,000 adults take the LSAT every year, this training program provided the opportunity to study plasticity in a real-world setting.

\section{Materials and Methods}

Participants. Research was approved by the Committee for the Protection of Human Subjects at the University of California at Berkeley. The training group included 26 prelaw adults ( 15 females), and the control group included 25 prelaw adults ( 15 females) who were age- and IQ-matched. Participants in the training group were recruited through an E-mail announcement and an in-class announcement to students in Blueprint Test Preparation courses. Participants in the control group were recruited through e-mails to prelaw organizations on campus and online postings.

Participants had no history of psychiatric or neurological disorder. All participants were fluent in English. Because our predictions for trainingrelated changes did not vary by handedness, we enrolled both right- and left-handed participants. In the training group, three participants were left-handed, and in the control group, two participants were left-handed. Two participants-one from the trained group and one from the control group-were excluded from the study based on dramatic change in stress levels and amount of sleep from time 1 to time 2 ( $>3$ SD from the mean of all participants). Additionally, one participant from the control group was excluded for excessive movement during the scan session $(>3 \mathrm{SD}$ from mean relative motion of all participants). Data are reported for 25 participants ( 14 females) in the trained group and 23 participants (14 females) in the control group (Table 1).

Training paradigm. The Blueprint Test Preparation course was selected as the training paradigm because it provided more classroom time than other programs: $100 \mathrm{~h}$ distributed across the three components of the LSAT ( $35 \mathrm{~h}$ for Logic Games, $35 \mathrm{~h}$ for Logical Reasoning, and $30 \mathrm{~h}$ for Reading Comprehension). "Logic Game" questions require test takers to integrate a series of rules to sequence or group a set of items. "Logical Reasoning" questions ask them to determine the logical flaw in an argument, identify an assumption, or choose a statement that would strengthen or weaken an argument. The remaining $30 \mathrm{~h}$ of class time were dedicated to "Reading Comprehension" questions that require test takers to interpret short passages of text. A recent LSAT exam can be found at http://www.lsac.org/jd/pdfs/SamplePTJune.pdf.

For the Logic Games section, students were taught to break down problems into the essential information and to use diagrams to represent and integrate rules. For the Logical Reasoning section, students were taught basic logic principles (such as modus ponens and modus tollens), as well as how to avoid common logical fallacies. Students attempted problems at home and then instructors worked through the problems in class, answering any questions students might have. Special attention was paid to keeping motivation levels high by making the content fun through relatable examples.

Blueprint course instructors administered four practice tests spread evenly throughout the course. LSAT practice test scores were provided
Table 2. Regions implicated in reasoning

\begin{tabular}{rll}
\hline & Region & Anatomical group \\
\hline 1 & Rostrolateral PFC & PFC \\
2 & Middle frontal gyrus & \\
3 & Inferior frontal gyrus & \\
4 & Precentral gyrus & Motor \\
5 & Caudate & Striatum \\
6 & Putamen & \\
7 & Superior parietal & \\
8 & Supramarginal gyrus \\
9 & Angular gyrus & \\
10 & Posterior parietal & \\
11 & Precuneus & \\
\hline
\end{tabular}

either by the participants or by the test preparation course with the consent of the participants. We report improvement from first to last practice test for the full LSAT score and for scores on the subtests. Additionally, we fit linear models to full LSAT scores across practice tests for each subject, and used the slope as an index of individual differences in training effects.

Behavioral measures. During the first testing session, we administered the Matrix Reasoning and Vocabulary subtests of the Wechsler Adult Scale of Intelligence (WASI) (Wechsler, 1999) to match the groups on IQ, as well as the Young Adult Self Report (Achenbach, 1990; 1997) to screen out participants who scored in the clinical range. Because the stress of studying for an important test has been associated with changes in fronto-parietal connectivity (Liston et al., 2009), we collected the Perceived Stress Scale (Cohen et al., 1983) at both time points. Further, because sleep deprivation can also alter prefrontal function (Walker and Stickgold, 2006), we asked all participants to report on their sleep habits at both visits.

Image acquisition. MRI scanning was performed on a Siemens 3T Trio at the Brain Imaging Center at the University of California at Berkeley. Participants underwent a series of anatomical scans, two fMRI tasks adapted from prior studies and an rs-fMRI scan. The first task (Wendelken and Bunge, 2010) was a transitive inference task that involved judgments of relative weights of colored balls. The second task (Bishop et al., 2008) involved selecting which of several sets of letters did not follow the same rule. The order of scans was fixed across participants and time points, to ensure that any differences between groups or time points could not be accounted for by order effects.

Here, we report data from the rs-fMRI scan. During the 6 min rs-fMRI scan, participants were asked to relax and remain awake while gradientecho EPI data were acquired ( $\mathrm{TR}=2000 \mathrm{~ms}, \mathrm{TE}=25 \mathrm{~ms}, 33$ axial slices, $2.0 \times 1.8 \times 3.0 \mathrm{~mm}$ voxels, no interslice gap, flip angle $=90^{\circ}$, field of view $=230 \mathrm{~mm}$ ).

Preprocessing. FMRI data were preprocessed and analyzed with FEAT (FMRI Expert Analysis Tool) Version 5.98, part of FSL (FMRIB's Software Library, www.fmrib.ox.ac.uk/fsl) (Smith et al., 2004; Woolrich et al., 2009). The following preprocessing steps were performed at the single-subject level: slice-timing correction; motion correction using MCFLIRT (Jenkinson et al., 2002); non-brain removal using BET (Smith et al., 2002); spatial smoothing using a Gaussian kernel of FWHM 5 mm; high-pass temporal filtering (Gaussian-weighted least-squares straight line fitting, with $\sigma=50.0 \mathrm{~s}$ ). Registration to high-resolution structural and standard space images was performed using FLIRT (Jenkinson and Smith, 2001; Jenkinson et al., 2002). Additionally, high-resolution structural images were segmented using FAST (Zhang et al., 2001) to obtain masks of white matter and CSF, which were then registered to the functional images. Time courses were extracted from these masks and from outside of the brain, and were used as covariates of no interest along with their temporal derivatives.

We sought to provide a comprehensive view of training-related changes in functional connectivity between brain regions involved in reasoning. Thus, we looked to a meta-analysis of neuroimaging studies of reasoning ability (Prado et al., 2011) to generate a list of 11 brain regions most often recruited during performance of reasoning tasks (Table 2). 
Anatomical regions of interest were created from the unthresholded Harvard-Oxford cortical and subcortical atlases distributed with FSL (Desikan et al., 2006) (http://www.cma. mgh.harvard.edu/fsl_atlas.html). We included bilateral frontal [RLPFC, MFG (middle frontal gyrus), IFG (inferior frontal gyrus) pars opercularis, and precentral gyrus], striatum (caudate and putamen), and parietal (superior parietal, angular gyrus, supramarginal gyrus, posterior parietal cortex, and precuneus) regions. We created the RLPFC region by excluding the medial portion of the frontopolar cortex atlas region $(|\mathrm{X}|<14)$. We refer to the superior lateral occipital cortex region as posterior parietal cortex because it encompasses a large portion of parietal cortex (see Fig. 3B). Regions were registered into native subject space. Average time courses were extracted for each region from the residuals of a GLM that included the covariates of no interest described above. Time courses were bandpass filtered $(0.008 \mathrm{~Hz}<f<0.08 \mathrm{~Hz})$. Finally, time course data were scrubbed for motion-related artifacts by removing volumes with $>0.5 \mathrm{~mm}$ of motion relative to the previous volume (Power et al., 2011).

Statistical analyses. Pearson correlations for each pair of regions were computed and Fischertransformed to produce normally distributed values ( $z$-scores). Paired $t$ tests comparing time 1 to time 2 data were performed for the trained group. Group $\times$ Time ANOVAs were conducted to test whether changes in the trained group were greater than variability in the control group. Finally, unpaired $t$ tests were conducted to exclude correlation pairs that differed between groups at time 1 from further analysis. A false discovery rate (FDR) procedure was used with an $\alpha$-level of $p<$

0.05 to correct for multiple comparisons (Benjamini and Hochberg, 1995).

To show the magnitude and direction of the correlation changes in the trained group, we created correlation change matrices for each hemisphere and for cross-hemisphere connections. Correlation changes were colored by number of SDs from the mean change, calculated across all correlation pairs and both groups (mean $=-0.0002, \mathrm{SD}=0.06$ ). Changes that were significant at $p<0.05$ for both the Group $\times$ Time ANOVA and the paired $t$ test were marked with an asterisk.

To visualize the spatial distribution of correlation changes, we calculated the number of significant increased and decreased connections for each region. Each pairwise correlation change was counted twice, once for each region in the pair, for a total of 36 changes. Cortical regions are projected onto the brain surface using CARET (Computerized Anatomical Reconstruction and Editing Toolkit 5, http://www.nitrc.org/projects/ caret) (Van Essen et al., 2001), and subcortical regions are shown on slices.

Finally, to examine brain-behavior correlations, we tested whether the slope of LSAT improvement was correlated with connectivity changes. We performed Pearson correlations between LSAT slope and connectivity change for each of the pairs of regions. To visualize these correlations, we took the approach described above of grouping regions into PFC, Motor, Striatum, and Parietal Cortex. We then identified the connections for which the degree of change in coupling related to the degree of behavioral improvement.

\section{Results}

Demographics and behavioral results

The trained group and the control group were well matched in terms of age, gender, IQ, and the number of days between scan sessions (Table 1). Additionally, the groups were matched on stress levels and amount of sleep, and neither group exhibited a change in either of these variables from time 1 to time 2. Because head motion has been shown to alter resting-state connectivity patterns (Van Dijk et al., 2012; Power et al., 2011), we confirmed that neither relative head displacement nor number of volumes with $>0.5 \mathrm{~mm}$ relative head displacement changed between time points for either group or differed between groups at either time point $(P$ values $>0.2)$.

Participants in the trained group for whom practice test scores were available $(n=21)$ improved significantly on the LSAT $(p<$ $0.001, \mathrm{df}=20, t=6.7)$ (Fig. $1 A)$. For a subset of participants $(n=$ 18 ), scores for each of the subtests were available (Fig. 1B). These scores revealed significant improvements on the two reasoning components of test, Logic Games $(p=0.001, \mathrm{df}=20, t=3.56)$ and Logical Reasoning $(p<0.001, \mathrm{df}=20, t=6.08)$. By contrast, there was no change in Reading Comprehension scores, although participants spent 30 classroom hours preparing for this section of the exam $(p=0.41, \mathrm{df}=17, t=0.84)$. The gains on the reasoning subtests were significantly greater than the change in Reading Comprehension (Logic Game: $p=0.004$, df $=17, t=$ 2.92; Logical Reasoning: $p=0.003, \mathrm{df}=17, t=3.16$ ).

The improvement in LSAT total score corresponds roughly, depending on the year, to an improvement from the $44^{\text {th }}$ percentile to the $73^{\mathrm{rd}}$ percentile. For a student with the mean grade point average of the trained group (3.4), a change from 149 to 157 would vastly widen the pool of law schools to which he or she had a realistic chance of acceptance (http://www.bc.edu/offices/careers/ gradschool/law/lawlocator.html). 


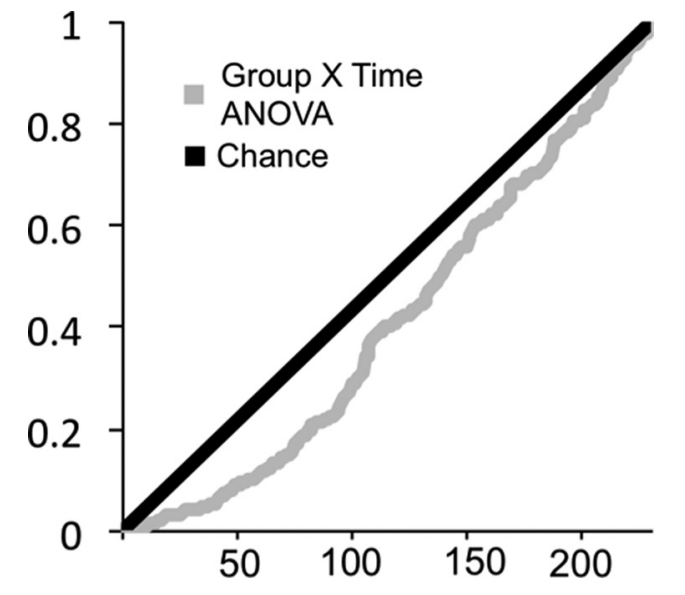

Figure 2. $P$-value distribution for Group $\times$ Time ANOVA. $P$-value distribution for Group $\times$ Time ANOVA of the 231 pairwise correlations, ordered from lowest to highest (black). The distribution expected by chance is marked by a gray line.

Scores for at least three practice LSAT tests were available for 20 subjects (Fig. 1C), making it possible to summarize performance changes by fitting a line to the practice test data (Fig. 1D). We calculated the slope of change for each subject based on their fitted line, and used these values to test for brain-behavior relationships in the magnitude of behavioral improvement and change in connectivity across subjects.

\section{rs-fMRI analyses}

We sought to provide a comprehensive view of training-related changes in functional connectivity between brain regions involved in reasoning. We extracted time courses from structural definitions of the 11 regions listed in Table 2 in each hemisphere, calculated pairwise correlations between regions, and Fishertransformed the correlations so as to be able to perform parametric statistics. We tested for an effect of training on each of the 231 pairwise correlations. The distribution of significance values ( $P$-values) for the 231 Group $\times$ Time ANOVAs deviated from chance, as shown in Figure 2. Forty-one pairs exhibited a $P$-value of $<0.05$ in the Group $\times$ Time ANOVA. Within the trained group, the distribution of $P$-values for time 1 to time $2 t$ tests also included many more low $P$-values than expected by chance: 51 pairs had a $P$-value of $<0.05$. In the control group, 22 pairs had a $\mathrm{P}$-value of $<0.05$. Small changes in the control group could be explained by increased familiarity with the scanner environment or maturational changes in the young adult brain. Table 3 shows detailed statistics for the 22 correlation changes that were significant in both the Group $\times$ Time ANOVA and the trained group paired $t$ test at $p<0.05$, and did not show a significant difference between groups at time 1 . Thus, Table 3 features the pairwise correlations that met the three conditions for a stringent test for training-related changes. All but one of the pairs that met these three conditions exhibited increased connectivity with training.

Overall, across groups and time points, the correlation values computed for these large anatomically defined ROIs were weak, compared with the values that one might expect for small, functionally defined ROIs: $84 \%$ of correlations fell between -0.3 and 0.3 . Of those correlations, $47 \%$ were positive and $53 \%$ were negative. Similarly, with respect to the changes in coupling observed in the trained group, $59 \%$ of the pairs that exhibited significant changes were weakly negatively correlated at time $1(-0.3<z<$ $0)$, and $36 \%$ were weakly positively correlated at time $1(0<z<$ 0.3 ). One connection, between left and right precentral gyri, was strongly positively correlated at time $1(z=0.85)$, and showed additional strengthening with training.

Having confirmed that the trained group exhibited a larger number of changed correlations than expected by chance, we sought to visualize the magnitude of change for each pair of regions for the trained group. We created correlation change matrices (Fig. 3) with brain regions ordered from anterior to posterior along each axis, following the order in Table 2. Pairs that met the stringent test for training-related changes (listed in Table 3) are marked with an asterisk. Within the left hemisphere, increased connections were observed between prefrontal and parietal cortices (Fig. 3Ai). A similar pattern of increased frontoparietal connectivity was seen between hemispheres (Fig. 3Aii). Within each hemisphere, and between hemispheres, we found increased connectivity between parietal cortices and the striatum (Fig. 3Aiii).

To visualize the regions that exhibited the greatest number of the increased correlations in Table 3, we color-coded each of the 11 regions per hemisphere according to the number of increased correlations involving that region (Fig. $3 B$ ). Here, we see that left precuneus exhibited five increased connections, and that left RLPFC, left angular gyrus, left posterior parietal cortex, and right putamen each exhibited four increased connections.

Next, to better visualize network changes for the trained group, we created a diagram (Fig. $4 A$ ) featuring connections between the four broad classes of regions in each hemisphere: PFC (including RLPFC, MFG, IFG), Motor (precentral gyrus), Striatum (caudate and putamen), and Parietal (superior parietal, angular gyrus, supramarginal gyrus, posterior parietal cortex, and precuneus). Figure $4 A$ highlights the prominence of changes involving left parietal cortex-in particular, an increase in the temporal coupling of this region with left and right prefrontal cortex and with right striatum.

Finally, we tested whether connectivity changes were related to slope in LSAT improvement for the 20 participants for whom practice test data were available. We observed 12 positive correlations between connectivity changes and LSAT improvement at $p<0.05$, uncorrected, where 6 would be expected by chance (Table 4; Fig. 4B). Nine of the 12 positive relationships involved the precuneus. Connectivity increases between left parietal and right PFC were most strongly associated with behavioral improvement, followed by increased connectivity between left striatum and right parietal (Fig. 4B). We also observed 6 negative brain-behavior correlations (Table 4; Fig. 4C), none of which showed a group effect of training (Fig. 4C).

\section{Discussion}

Broadly, we sought to test the hypothesis that engaging in novel, complex cognitive tasks would strengthen connectivity within the reasoning network at rest. Indeed, the present findings show that connectivity between brain areas implicated in cognition is experience dependent and can be modified by intensive training.

More specifically, we predicted that relational reasoning training would strengthen connectivity between RLPFC, a region implicated in relational integration, and parietal regions thought to support processing of individual relations. We found increased frontoparietal connectivity, primarily within the left hemisphere and between hemispheres. As predicted, left RLPFC showed many correlation increases, specifically with posterior and medial parietal regions. In addition to the increase in fronto-parietal coupling, we observed an increase in connectivity between parietal cortex and the striatum. These changes were significant both between and within hemispheres. While not predicted in ad- 
Table 3. Correlation changes with training

\begin{tabular}{|c|c|c|c|c|c|c|c|}
\hline Region 1 & Region 2 & Change & Trained Time $1 z$ & Trained Time $2 z$ & Trained $t$ test & Control $t$ test & $\overline{\text { ANOVA }}$ \\
\hline LRLPFC & $\mathrm{R}$ Precuneus & + & -0.26 & -0.09 & 0.0005 & 0.41 & 0.01 \\
\hline L Precentral & $\mathrm{R}$ Precentral & + & 0.85 & 1.04 & 0.0007 & 0.85 & 0.02 \\
\hline R Putamen & L Angular & + & -0.05 & 0.03 & 0.001 & 0.71 & 0.05 \\
\hline R Caudate & L Angular & + & -0.01 & 0.09 & 0.001 & 0.68 & 0.02 \\
\hline L Posterior Parietal & RIFG & + & -0.15 & -0.02 & 0.002 & 0.10 & 0.001 \\
\hline R Putamen & $\mathrm{R}$ Precuneus & + & -0.16 & -0.05 & 0.002 & 0.46 & 0.01 \\
\hline LRLPFC & L Precuneus & + & -0.08 & 0.08 & 0.002 & 0.74 & 0.03 \\
\hline R Putamen & L Post Parietal & + & -0.19 & -0.07 & 0.003 & 0.67 & 0.01 \\
\hline L Angular & RIFG & + & 0.01 & 0.13 & 0.01 & 0.83 & 0.03 \\
\hline L Posterior Parietal & LIFG & + & 0.04 & 0.14 & 0.01 & 0.16 & 0.005 \\
\hline L Putamen & L Precuneus & + & -0.14 & -0.05 & 0.01 & 0.41 & 0.02 \\
\hline L Caudate & L Precuneus & + & -0.13 & -0.01 & 0.01 & 0.83 & 0.05 \\
\hline RRLPFC & L Angular & + & 0.08 & 0.18 & 0.01 & 0.22 & 0.01 \\
\hline R Putamen & R Angular & + & -0.01 & 0.07 & 0.02 & 0.54 & 0.05 \\
\hline R Caudate & L Precuneus & + & -0.14 & -0.04 & 0.02 & 0.49 & 0.02 \\
\hline L Caudate & R Angular & + & 0.03 & 0.12 & 0.02 & 0.55 & 0.03 \\
\hline RIFG & L Precentral & - & 0.08 & 0.00 & 0.02 & 0.36 & 0.05 \\
\hline LRLPFC & R Angular & + & 0.09 & 0.19 & 0.03 & 0.55 & 0.04 \\
\hline L Precuneus & LIFG & + & -0.16 & -0.06 & 0.03 & 0.07 & 0.005 \\
\hline R Posterior Parietal & RIFG & + & -0.04 & 0.06 & 0.04 & 0.10 & 0.01 \\
\hline L Supramarginal & L Post Parietal & + & 0.07 & 0.15 & 0.04 & 0.30 & 0.03 \\
\hline L Caudate & LRLPFC & + & 0.29 & 0.40 & 0.04 & 0.47 & 0.04 \\
\hline
\end{tabular}

Detailed statistics for correlation changes that were significant at $p<0.05$ for both the Group $\times$ Time ANOVA and the paired $t$ test for the trained group. Three correlation pairs that were significantly different between groups at time 1 were removed from further analysis. Bolded $P$-values survived an FDR correction for multiple comparisons.

\section{A Pairwise Correlation Changes}

\section{i. Ipsilateral: Left-Left}
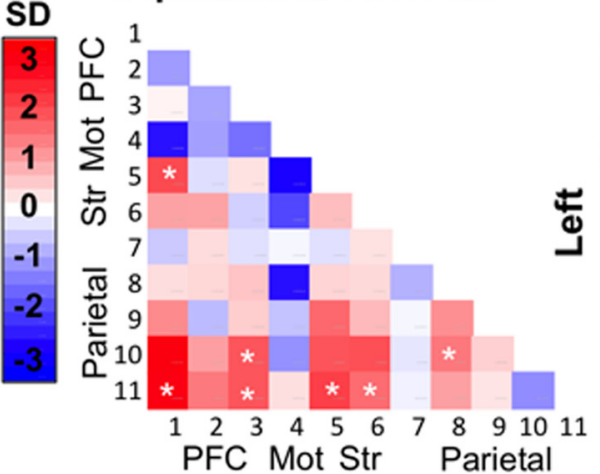

ii. Contralateral

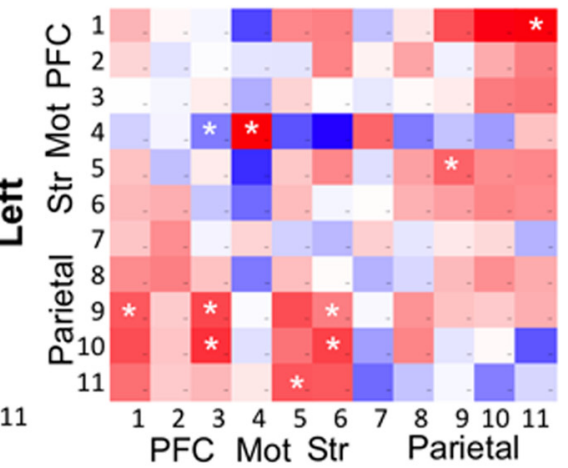

iii. Ipsilateral: Right-Right

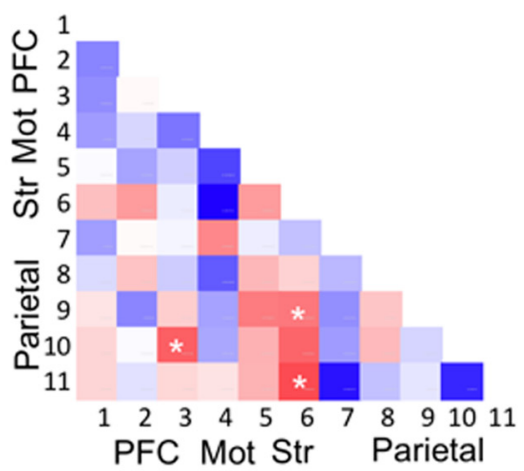

Right

\section{B Regions Showing Correlation Changes}

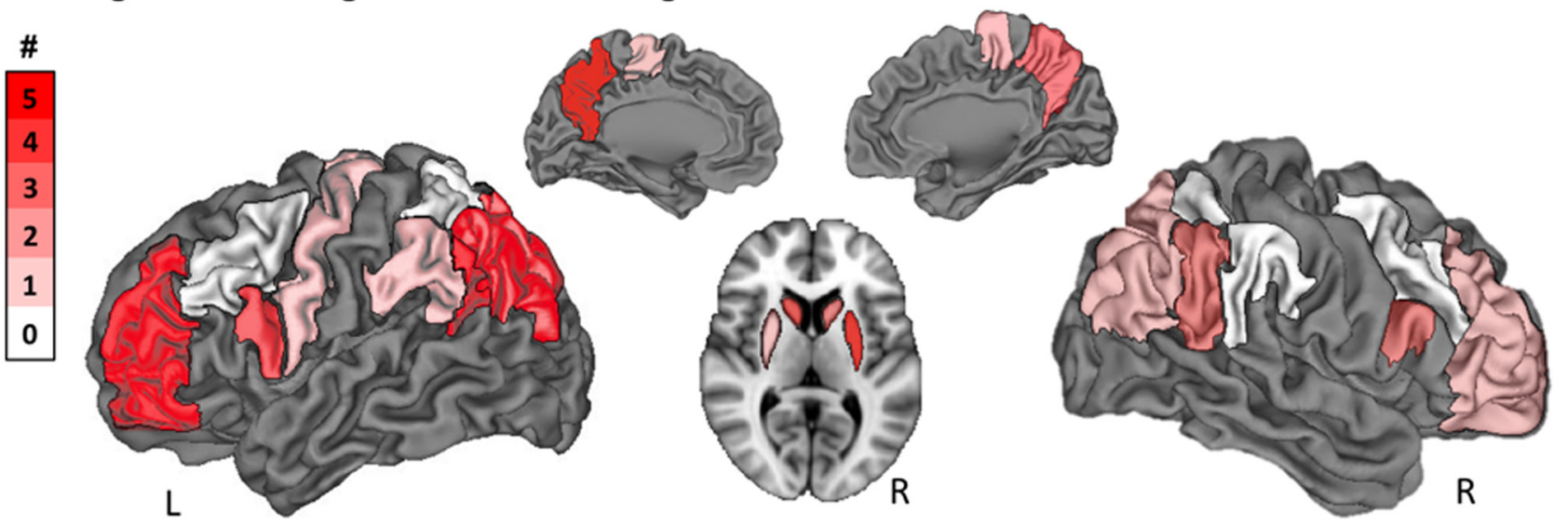

Figure 3. Changes in pairwise correlations. A, Magnitude of change for all pairwise correlations. Correlations are colored by SDs from the mean calculated across all pairs and both groups. Region numbers refer to Table 2. PFC, Prefrontal cortex; Mot, motor cortex; Str, striatum. White asterisks denote significant changes (see Table 2). $i$, Connections within the left hemisphere. ii, Connections between the left and right hemispheres. iii, Connections within the right hemisphere). $\boldsymbol{B}$, Number of increased correlations for each region (see Table 3 ). Regions were taken from the Harvard-0xford Atlas and rendered on a CARET brain. 


\section{A Connection Changes with Training}

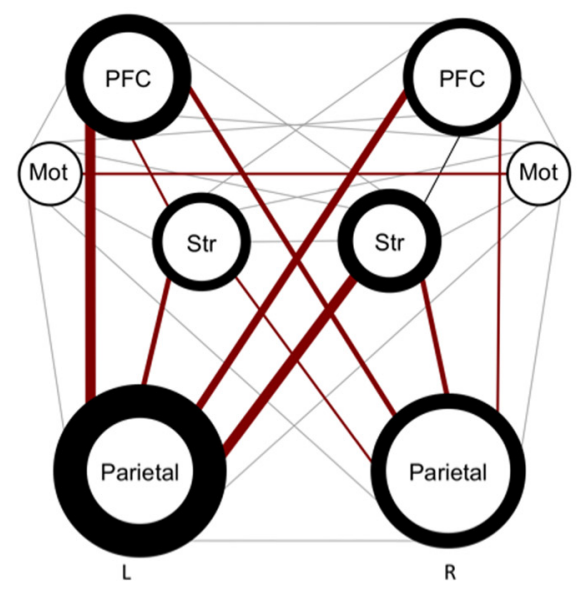

\section{B Positive Correlations with LSAT Improvement}

\section{Negative Correlations with LSAT Improvement}
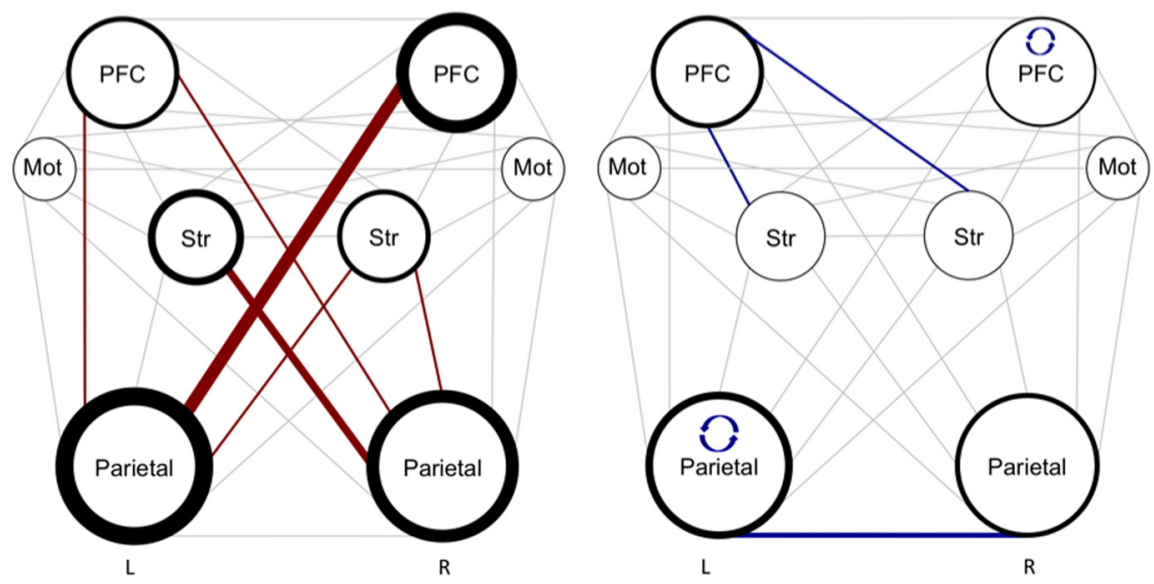

Figure 4. Connectivity changes and correlations with LSAT improvement. These diagrams show training-related increases in connectivity at the group level $(\boldsymbol{A})$ and individual differences in connectivity changes that were related to behavioral improvement $(\boldsymbol{B}, \boldsymbol{C})$. $\boldsymbol{A}$, The size of the circle representing each anatomical group reflects the number of regions it includes. The weight of the line around each circle reflects the number of changed connections involving regions within the anatomical group. The weight of the line anatomical groups represents the number of changed connections between them (i.e., between the regions included in each anatomical group). Note that one significant decrease in connectivity is not shown (RPFC to L Mot). $\boldsymbol{B}, \boldsymbol{C}$, Positive (B) and negative (C) relationships between LSAT slope (Fig. $1 \boldsymbol{D})$ and connectivity change. The weight of the line around a circle represents the number of connections involving that anatomical group that were correlated with behavior. The weight of the connecting lines between anatomical groups represents the number of connections between them that exhibited brain- behavior correlations. The reciprocal curved arrows shown in C indicate that correlations among regions within left parietal cortex and within right PFC were negatively correlated with LSAT improvement.

vance, the increase in parieto-striatal connectivity is consistent with the known role of the striatum in reasoning (Melrose et al., 2007; Prado et al., 2011), as well as in skill learning across both cognitive and motor domains, based on its strong dopaminergic inputs (Ashby et al., 2010).

When we tested whether observed changes in connectivity were linked to behavioral improvement, we found that frontoparietal and parieto-striatal connection changes were positively correlated with LSAT improvement. However, none of these brain-behavior correlations survived a correction for multiple comparisons. It should be noted that the behavioral measure (the LSAT) was designed for a real-world purpose rather than for perfect psychometric validity and reliability, and also that the brain and behavior measures were collected on different days.

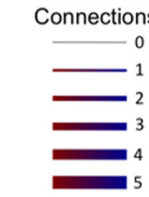

Anatomical Group

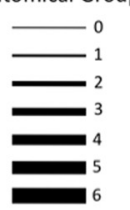

These findings serve as a proof of concept that 3 months' practice of reasoning skills alters fronto-parietal connectivity, with hints of individual differences in the magnitude of neural changes reflecting differences in the magnitude of performance changes. This proof-of-concept study should be followed up with a second study with a large enough sample size to measure and control for possible sources of interindividual variability, including gender, age, differences in pretraining reasoning abilities, and types of activities pursued during the training period.

A potential caveat is that the restingstate data were acquired during the same scan session as task data. Indeed, other studies have shown that these networks can be altered over the short term by preceding the rs-fMRI scan with a task (Waites et al., 2005; Harrison et al., 2008; Stevens et al., 2010). However, since the order of scans was fixed across groups and time points, it is unlikely that the tasks influenced resting-state data in a systematic way that was specific to the trained group. Further, the tasks performed before the rs-fMRI scan bore little resemblance to the types of problems students practiced during the LSAT training. Finally, we have recently demonstrated training-related changes in white matter microstructure in fronto-frontal and frontoparietal tracts for the same study sample (Mackey et al., 2012). Thus, the most parsimonious explanation for the observed changes in functional connectivity is that 3 months of cognitive training altered the strength of coordinated activity within and across large-scale brain networks.

While it is possible only to speculate about the cellular processes underlying changes in functional connectivity in humans, structural MRI data could shed light on which mechanisms may be at work. The training-related changes in diffusion parameters that we have observed for the LSAT group (Mackey et al., 2012) could indicate a change in myelination, axon coherence, and/or properties of astrocytes (Zatorre et al., 2012). Computational modeling may be needed to fully explain how the documented changes in functional connectivity in the lateral frontoparietal network relate to the changes in white matter microstructure within this network.

Not only do the observed changes in resting-state connectivity speak to the functional roles of the regions involved, but they also challenge the notion that resting-state networks supporting higher cognition are stable in adulthood. These results build on evidence of training-induced resting-state changes in other domains, including motor learning (Taubert et al., 2011), processing speed (Takeuchi et al., 2011), and meditation (Xue et al., 2011). Additionally, there is preliminary evidence that working 
Table 4. Correlations between connectivity changes and LSAT improvement

\begin{tabular}{llrl}
\hline Region 1 & Region 2 & $r$ & $p$ \\
\hline Positive correlations & & & \\
L RLPFC & R Precuneus & 0.52 & 0.02 \\
R RLPFC & L Precuneus & 0.52 & 0.02 \\
L Caudate & R Precuneus & 0.51 & 0.02 \\
R Caudate & R Precuneus & 0.51 & 0.02 \\
L Posterior Parietal & R IFG & 0.50 & 0.02 \\
L RLPFC & L Precuneus & 0.47 & 0.03 \\
L Precuneus & R MFG & 0.47 & 0.03 \\
L Putamen & R Precuneus & 0.46 & 0.04 \\
L Caudate & R Post Parietal & 0.45 & 0.04 \\
L Posterior Parietal & R MFG & 0.45 & 0.04 \\
L Precuneus & R IFG & 0.45 & 0.04 \\
R Caudate & L Precuneus & 0.44 & 0.05 \\
Negative correlations & & & \\
R RLPFC & R MFG & -0.59 & 0.006 \\
L Supramarginal & L Angular & -0.55 & 0.01 \\
R Caudate & L MFG & -0.52 & 0.02 \\
L Angular & R Angular & -0.49 & 0.02 \\
R Supramarginal & L Angular & -0.49 & 0.03 \\
L Caudate & L RLPFC & -0.45 & 0.04 \\
\hline
\end{tabular}

Table is ordered from lowest to highest $P$-value separately for positive and negative behavioral correlations.

memory training may also alter resting-state connectivity (Jolles et al., 2013). Demonstrating neural plasticity in the network that supports reasoning - a skill that is central to theories of intelligence (Gray and Thompson, 2004)—is particularly significant because it runs counter to the widespread assumption that intelligence is a fixed ability.

The results of the current study have broad societal relevance. Millions of young adults prepare intensively for the LSAT and other standardized exams. To correctly interpret the significance of these test scores, it is important to know whether these exams measure individuals' cognitive potential, or whether they more accurately reflect their cognitive history-i.e., the prior level of engagement of specific brain networks.

Finally, understanding plasticity in cognitive skills in healthy adults is critical as more and more people extend their formal education into the third and fourth decades of their lives. More broadly, throughout the lifespan, individuals encounter profound shifts in their environments that necessitate categorical changes in cognition. Neural plasticity at the synaptic level, and indeed at the level of large-scale networks, enables our brains to rise to meet novel cognitive demands. However, changes in brain connectivity associated with a brief change in cognitive activity are unlikely to last indefinitely, just as a student who has just prepared intensively for the LSAT is unlikely to perform as well on the exam after many months have elapsed. To maintain a high level of reasoning ability, we hypothesize, it is important to regularly tax the underlying brain circuitry.

\section{References}

Achenbach T (1990) Young Adult Self Report. Burlington, VT: University of Vermont, Department of Psychiatry.

Achenbach T (1997) Young Adult Self Report. Burlington, VT: University of Vermont, Department of Psychiatry.

Ashby FG, Turner BO, Horvitz JC (2010) Cortical and basal ganglia contributions to habit learning and automaticity. Trends Cogn Sci 14:208-215. CrossRef Medline

Bao S, Chan VT, Merzenich MM (2001) Cortical remodelling induced by activity of ventral tegmental dopamine neurons. Nature 412:79-83. CrossRef Medline

Benjamini Y, Hochberg Y (1995) Controlling the false discovery rate-a practical and powerful approach to multiple testing. J R Stat Soc Ser B Methodol 57:289-300.
Bergan JF, Ro P, Ro D, Knudsen EI (2005) Hunting increases adaptive auditory map plasticity in adult barn owls. J Neurosci 25:9816-9820. CrossRef Medline

Bishop SJ, Fossella J, Croucher CJ, Duncan J (2008) COMT val158met genotype affects recruitment of neural mechanisms supporting fluid intelligence. Cereb Cortex 18:2132-2140. Medline

Cohen S, Kamarck T, Mermelstein R (1983) A global measure of perceived stress. J Health Soc Behav 24:385-396. CrossRef Medline

Desikan RS, Segonne F, Fischl B, Quinn BT, Dickerson BC, Blacker D, Buckner RL, Dale AM, Maguire RP, Hyman BT, Albert MS, Killiany RJ (2006) An automated labeling system for subdividing the human cerebral cortex on MRI scans into gyral based regions of interest. Neuroimage 31:968980. CrossRef Medline

Dosenbach NU, Fair DA, Miezin FM, Cohen AL, Wenger KK, Dosenbach RA, Fox MD, Snyder AZ, Vincent JL, Raichle ME, Schlaggar BL, Petersen SE (2007) Distinct brain networks for adaptive and stable task control in humans. Proc Natl Acad Sci U S A 104:11073-11078. CrossRef Medline

Fair DA, Cohen AL, Power JD, Dosenbach NU, Church JA, Miezin FM, Schlaggar BL, Petersen SE (2009) Functional brain networks develop from a "local to distributed" organization. PLoS Comput Biol 5:e1000381. CrossRef Medline

Ferrer E, O'Hare ED, Bunge SA (2009) Fluid reasoning and the developing brain. Front Neurosci 3:46-51. Medline

Fox MD, Raichle ME (2007) Spontaneous fluctuations in brain activity observed with functional magnetic resonance imaging. Nat Rev Neurosci 8:700-711. CrossRef Medline

Fu M, Zuo Y (2011) Experience-dependent structural plasticity in the cortex. Trends Neurosci 34:177-187. CrossRef Medline

Gray JR, Thompson PM (2004) Neurobiology of intelligence: science and ethics. Nat Rev Neurosci 5:471-482. CrossRef Medline

Halford GS, Wilson WH, Phillips S (1998) Processing capacity defined by relational complexity: implications for comparative, developmental, and cognitive psychology. Behav Brain Sci 21:803-831. Medline

Harrison BJ, Pujol J, Ortiz H, Fornito A, Pantelis C, Yucel M (2008) Modulation of brain resting-state networks by sad mood induction. PLoS One 3:e1794. CrossRef Medline

Hummel JE, Holyoak KJ (2005) Relational reasoning in a neurally plausible cognitive architecture. Curr Dir Psychol Sci 14:153-157. CrossRef

Jenkinson M, Smith S (2001) A global optimisation method for robust affine registration of brain images. Med Image Anal 5:143-156. CrossRef Medline

Jenkinson M, Bannister P, Brady M, Smith S (2002) Improved optimization for the robust and accurate linear registration and motion correction of brain images. Neuroimage 17:825-841. CrossRef Medline

Jolles DD, van Buchem MA, Crone EA, Rombouts SA (2013) Functional brain connectivity at rest changes after working memory training. Hum Brain Mapp 34:396-406. CrossRef Medline

Krawczyk DC (2012) The cognition and neuroscience of relational reasoning. Brain Res 1428:13-23. CrossRef Medline

Liston C, McEwen BS, Casey BJ (2009) Psychosocial stress reversibly disrupts prefrontal processing and attentional control. Proc Natl Acad Sci U S A 106:912-917. CrossRef Medline

Mackey AP, Whitaker KJ, Bunge SA (2012) Experience-dependent plasticity in white matter microstructure: reasoning training alters structural connectivity. Front Neuroanat 6:32. CrossRef Medline

Mars RB, Jbabdi S, Sallet J, O’Reilly JX, Croxson PL, Olivier E, Noonan MP, Bergmann C, Mitchell AS, Baxter MG, Behrens TE, Johansen-Berg H, Tomassini V, Miller KL, Rushworth MF (2011) Diffusion-weighted imaging tractography-based parcellation of the human parietal cortex and comparison with human and macaque resting-state functional connectivity. J Neurosci 31:4087-4100. CrossRef Medline

Melrose RJ, Poulin RM, Stern CE (2007) An fMRI investigation of the role of the basal ganglia in reasoning. Brain Res 1142:146-158. CrossRef Medline

Nelson SM, Cohen AL, Power JD, Wig GS, Miezin FM, Wheeler ME, Velanova K, Donaldson DI, Phillips JS, Schlaggar BL, Petersen SE (2010) A parcellation scheme for human left lateral parietal cortex. Neuron 67: 156-170. CrossRef Medline

Power JD, Barnes KA, Snyder AZ, Schlaggar BL, Petersen SE (2011) Spurious but systematic correlations in functional connectivity MRI networks arise from subject motion. Neuroimage 59:2142-2154.

Prado J, Chadha A, Booth JR (2011) The brain network for deductive rea- 
soning: a quantitative meta-analysis of 28 neuroimaging studies. J Cogn Neurosci 23:3483-3497. CrossRef Medline

Seeley WW, Menon V, Schatzberg AF, Keller J, Glover GH, Kenna H, Reiss AL, Greicius MD (2007) Dissociable intrinsic connectivity networks for salience processing and executive control. J Neurosci 27:2349-2356. CrossRef Medline

Shehzad Z, Kelly AM, Reiss PT, Gee DG, Gotimer K, Uddin LQ, Lee SH, Margulies DS, Roy AK, Biswal BB, Petkova E, Castellanos FX, Milham MP (2009) The resting brain: unconstrained yet reliable. Cereb Cortex 19: 2209-2229. CrossRef Medline

Smith SM, Zhang Y, Jenkinson M, Chen J, Matthews PM, Federico A, De Stefano N (2002) Accurate, robust, and automated longitudinal and cross-sectional brain change analysis. Neuroimage 17:479-489. CrossRef Medline

Smith SM, Jenkinson M, Woolrich MW, Beckmann CF, Behrens TE, Johansen-Berg H, Bannister PR, De Luca M, Drobnjak I, Flitney DE, Niazy RK, Saunders J, Vickers J, Zhang Y, De Stefano N, Brady JM, Matthews PM (2004) Advances in functional and structural MR image analysis and implementation as FSL. Neuroimage 23 [Suppl 1]:S208-S219.

Stevens WD, Buckner RL, Schacter DL (2010) Correlated low-frequency BOLD fluctuations in the resting human brain are modulated by recent experience in category-preferential visual regions. Cereb Cortex 20:1997-2006. CrossRef Medline

Takeuchi H, Taki Y, Hashizume H, Sassa Y, Nagase T, Nouchi R, Kawashima $\mathrm{R}$ (2011) Effects of training of processing speed on neural systems. J Neurosci 31:12139-12148. CrossRef Medline

Taubert M, Lohmann G, Margulies DS, Villringer A, Ragert P (2011) Longterm effects of motor training on resting-state networks and underlying brain structure. Neuroimage 57:1492-1498. CrossRef Medline

Van Dijk KR, Sabuncu MR, Buckner RL (2012) The influence of head motion on intrinsic functional connectivity MRI. Neuroimage 59:431-438. CrossRef Medline
Van Essen DC, Drury HA, Dickson J, Harwell J, Hanlon D, Anderson CH (2001) An integrated software system for surface-based analyses of cerebral cortex. J Am Med Inform Assoc 8:443-459. CrossRef Medline

Waites AB, Stanislavsky A, Abbott DF, Jackson GD (2005) Effect of prior cognitive state on resting state networks measured with functional connectivity. Hum Brain Mapp 24:59-68. CrossRef Medline

Walker MP, Stickgold R (2006) Sleep, memory, and plasticity. Annu Rev Psychol 57:139-166. CrossRef Medline

Wechsler D (1999) Wechsler Abbreviated Scale of Intelligence. San Antonio, TX: The Psychological Corporation.

Wendelken C, Bunge SA (2010) Transitive inference: distinct contributions of rostrolateral prefrontal cortex and the hippocampus. J Cogn Neurosci 22:837-847. CrossRef Medline

Wendelken C, O'Hare ED, Whitaker KJ, Ferrer E, Bunge SA (2011) Increased functional selectivity over development in rostrolateral prefrontal cortex. J Neurosci 31:17260-17268. CrossRef Medline

Wendelken C, Chung D, Bunge SA (2012) Rostrolateral prefrontal cortex: domain-general or domain-sensitive? Hum Brain Mapp 33:1952-1963. CrossRef

Woolrich MW, Jbabdi S, Patenaude B, Chappell M, Makni S, Behrens T, Beckmann C, Jenkinson M, Smith SM (2009) Bayesian analysis of neuroimaging data in FSL. Neuroimage 45:S173-S186. CrossRef Medline

Xue S, Tang YY, Posner MI (2011) Short-term meditation increases network efficiency of the anterior cingulate cortex. Neuroreport 22:570 -574. Medline

Zatorre RJ, Fields RD, Johansen-Berg H (2012) Plasticity in gray and white: neuroimaging changes in brain structure during learning. Nat Neurosci 15:528-536. CrossRef Medline

Zhang Y, Brady M, Smith S (2001) Segmentation of brain MR images through a hidden Markov random field model and the expectation maximization algorithm. IEEE Trans Med Imaging 20:45-57. CrossRef Medline 